\title{
Temporada reproductiva del lobo fino austral, Arctocephalus australis (Zimmerman, 1783) en la Isla Guafo, Chiloé, Chile
}

\author{
Breeding season of the southern fur seal, Arctocephalus australis at Guafo Island, \\ southern Chile
}

HÉCTOR J. PAVÉS ${ }^{1 *} \&$ ROBERTO P. SCHLATTER ${ }^{1}$

${ }^{1}$ Instituto de Zoología, Universidad Austral de Chile, Casilla 567, Valdivia, Chile

*e-mail para correspondencia: hpaves@surnet.cl

\begin{abstract}
RESUMEN
A lo largo del litoral sudamericano se registran ejemplares del lobo fino austral (Arctocephalus australis), especie que al establecerse en una amplia área de distribución, presentaría una marcada sincronización reproductiva y un fuerte efecto latitudinal sobre sus patrones reproductivos. En este trabajo se revisarán los patrones reproductivos de A. australis en Isla Guafo $\left(43^{\circ} 33^{\circ} \mathrm{S}, 74^{\circ} 51^{\prime} \mathrm{O}\right.$, Chile), determinando existencia de una sincronización y estabilidad temporal en los partos, cópulas y en la estacionalidad de los territorios durante dos temporadas reproductivas consecutivas. Se determinó la presencia de machos territoriales desde mediados de noviembre a principios de febrero, registrándose el máximo para ambas temporadas, durante la cuarta semana de diciembre. El periodo de sincronización de pariciones, se estimó entre la primera y cuarta semana de diciembre con una duración promedio de 28,5 $\pm 2,1$ días. La fecha media de partos, se estimó el día 15,5 de diciembre. La fecha media de cópulas se registró el día $19 \pm 1,4$ de diciembre con un periodo de sincronización de $29 \pm$ 4,2 días de duración. Empleando la fecha media de cópulas y partos, se estimó un ciclo reproductivo cercano a 11 meses y 27 días para la especie en Guafo. Solo se determinaron diferencias estadísticamente significativas para la fecha del máximo de partos entre temporadas, no así para las cópulas ni la estacionalidad de los territorios. Fue posible determinar estabilidad en el tamaño poblacional y estructura etaria, no así en el índice de poliginia HPMT. Aunque se determinó una diferencia estadística en la fecha de pariciones, fue posible identificar una marcada estacionalidad y sincronización reproductiva para la especie (cerca de 30 días). Conjuntamente, se determinó que los patrones reproductivos evaluados presentaron una estabilidad temporal en el sector, condición compartida con el resto de Otáridos de ambientes templados. Mediante comparación de los resultados obtenidos, con aquellas presentados para Sudamérica, se evidenciaron diferencias cercanas a 30 días en la fecha de partos, cópulas y establecimiento de los territorios. Esta información debe ser actualizada con urgencia para entender con ayuda de las herramientas filogeográficas la historia ecológica y evolutiva de esta especie en el Neotrópico.
\end{abstract}

Palabras clave: Arctocephalus australis, patrones reproductivos, sincronización reproductiva, poliginia.

\section{ABSTRACT}

The southern fur seal (Arctocephalus australis) has colonised an ample geographic coverage in South America. The population should present there reproductive synchronization and a strong latitudinal effect on its reproductive activities. This research intends to review those reproductive activities on Guafo island $\left(43^{\circ} 33^{\prime} \mathrm{S}, 74^{\circ} 51^{\prime} \mathrm{W}\right.$, Chile) looking after the existence of synchronization and stability during successive breeding seasons, considering birthing, mating and territorial seasonality. During both seasons we observed territorial males from the end of November to beginning of February, counting most of them during the 4th week in December. The period of synchronization for births was registered between the first and fourth week of December with a mean duration of $28.5 \pm 2.1$ days. The average maximum date for birthing on Guafo Island was estimated for the day 15,5 of December. The period of mating synchronization was registered on a similar date to the birthing. However, the mean date of mating was estimated to be day $19 \pm 1.4$ during December with a synchronization period of $29 \pm 4.2$ days of duration. By using the average mating and birth dates we estimated the reproductive cycle in about 11 months 27 days for the species at Guafo. We only determined statistically significant differences for the date of maximum birth between seasons, not for mating neither for the seasonality of territories. We could determine stability for the population size and age cohorts but not for the poliginic index HPMT. Even when we obtained statistical differences for the birth dates, we could identify a marked seasonality and reproductive synchronization for the species (about 30 days). At the 
same time we could determine that the evaluated reproductive patterns presented a temporal stability in the region, a condition shared with the rest of the Otariids of temperate environments. By comparing results with those presented for other sites in South America we evidenced differences in about 30 days for the dates of birthing, mating and territory establishments. This information has to be actualized as soon as possible for understanding with actual filogeographic tools the ecological history and evolution of this species in the Neotropics.

Key words: Arctocephalus australis, reproductive patterns, reproductive synchronization, polygyny.

\section{INTRODUCCION}

Al igual que todos los integrantes de la familia Otariidae, el lobo fino austral (Arctocephalus australis, Zimmerman, 1783) desarrolla sus actividades reproductivas a mediados de las temporadas estivales, agrupándose en lugares específicos donde conforma colonias reproductivas (Sielfeld 1983, Riedman 1990). En estos lugares se registra tanto un incremento de las actividades sociales (e.g., territorialidad, cuidado parental) como el desarrollo de eventos reproductivos no observadas durante el resto del año (e.g., pariciones, estro; Vaz-Ferreira 1965, Campagna 1985, Sielfeld 1999, Acevedo et al. 2003, Pavés et al. 2005a). Todo este ambiente generado, es potenciado por la presencia de condiciones ambientales favorables para el desarrollo y la supervivencia de la descendencia (i.e., meteorológicas, alimentarias) (Bowen 1991, Majluf 1992, Trites \& Antonelis 1994, Testa 2002).

El lobo fino austral es una especie que presenta un amplio rango de distribución latitudinal en el litoral de América del Sur, sin embargo, se identifican ejemplares en puntos extremos de su área de distribución (i.e., Perú, sur de Chile, Uruguay y Patagonia Argentina; Sielfeld 1983, Torres \& Contreras 1987, FAO 1993, Sielfeld 1999). En especies con amplias áreas de distribución, Riedman (1990) y Boness (1991), han mencionado la existencia de un efecto latitudinal sobre los eventos reproductivos entre sus poblaciones distantes. Este efecto se evidenciaría en una relación negativa entre el grado de sincronización reproductiva y la latitud donde se establecen las poblaciones o especies (Boness 1991). La relación sincronización reproductiva-latitud ha sido corroborada tanto para otros Otariidae como Phocidae (Riedman 1990, Campagna et al. 1993, Temte 1993, Goldsworthy \& Shaughnessy 1994, Pitcher et al. 2001, GarcíaAguilar \& Aurioles-Gamboa 2003), aproximación que evidenciaría una relación mayor entre ciclo reproductivo de cada especie/población con las características ambientales locales (Bowen 1991, Costa \& Gales 2003).

Aunque Boness (1991) describe este efecto tanto a nivel interespecífico como intraespecífico, los patrones reproductivos son identificados durante el mismo período de tiempo año tras año en ausencia de perturbaciones ambientales drásticas (e.g., El ENSO; Campagna 1985, Majluf 1992, Trites 1992, Goldsworthy \& Shaughnessy 1994, Acevedo et al. 2003, García-Aguilar \& Aurioles-Gamboa 2003, Soto et al. 2004, Pavés et al. 2005a). En este sentido, y al igual que lo registrado en congéneres del lobo fino austral (Arctocephalus australis), se esperaría que en esta especie las pariciones, cópulas y la formación de territorios se identifiquen durante un mismo y restringido periodo de tiempo año tras año. Inserto en este contexto, la presente contribución tiene por objeto describir la estación reproductiva del lobo fino austral en el sur de Chile, evaluando la sincronización y estabilidad de los patrones reproductivos durante dos temporadas reproductivas sucesivas. Además, se pretende revisar el conocimiento de los patrones reproductivos en América del Sur, información que en su conjunto permitirá iniciar el entendimiento de la ecología reproductiva de estos animales, básico para el desarrollo de adecuados planes de manejo y conservación para la especie (Sielfeld 1999).

\section{MATERIALES Y MÉTODOS}

Los muestreos fueron realizados en la colonia reproductiva del lobo fino austral, Arctocephalus australis, ubicada en el sector Punta Weather de la Isla Guafo (43 $33^{\prime}$ S, 74²9’ W, Chiloé, Chile) (Fig. 1). 


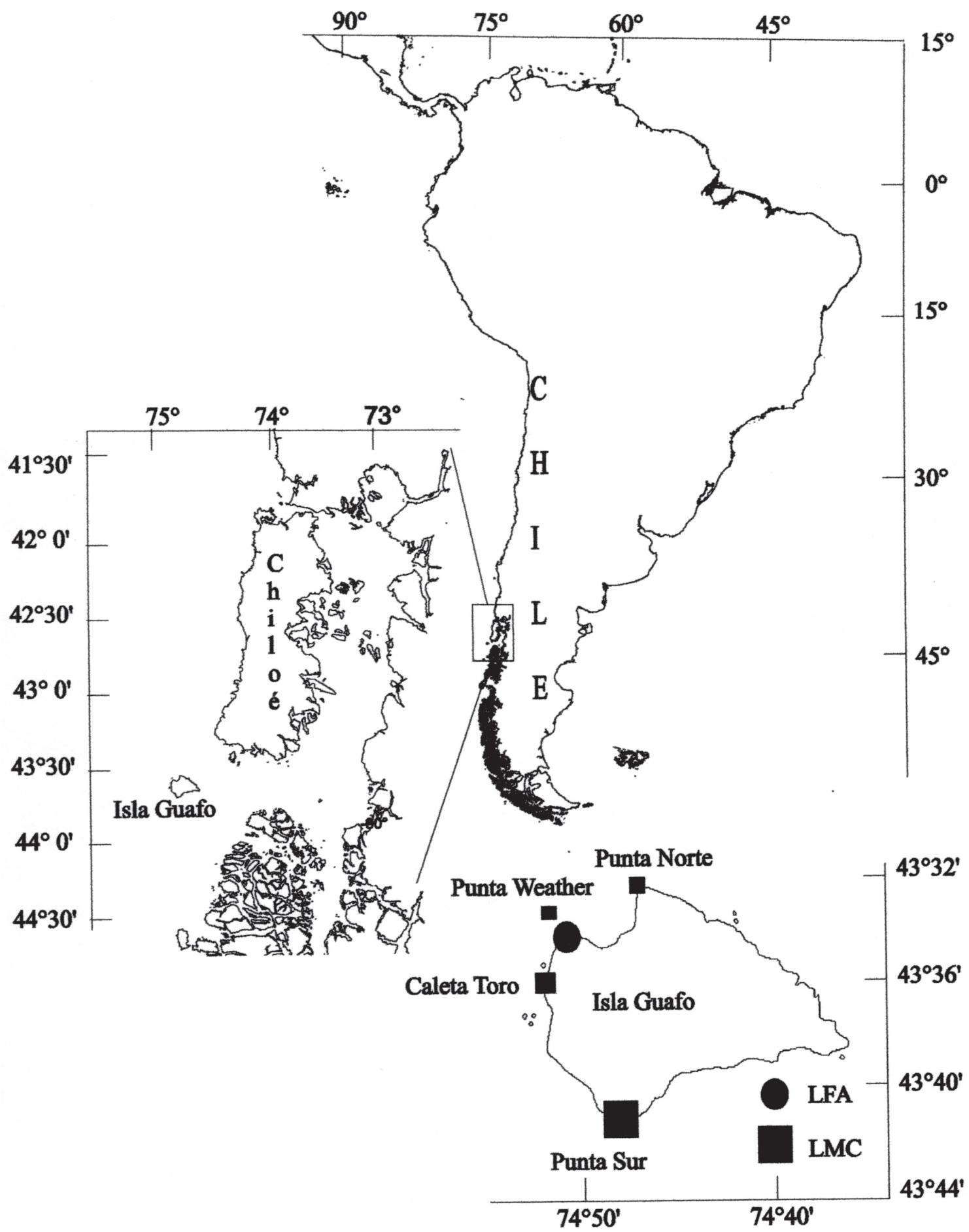

Fig. 1: Ubicación geográfica de Punta Weather (43 $33^{\prime} 57^{\prime}$ ' $\mathrm{S} ; 74^{\circ} 49^{\prime} 4^{\prime}$ ' 'W) en la Isla Guafo, Chiloé, Boca del Guafo, Canal Corcovado, X Región de Chile. Se indican la ubicación de la colonia reproductiva del lobo fino austral (LFA) y las del lobo marino común (LMC).

Geographical location of Punta Weather ( $43^{\circ} 33^{\prime} 57^{\prime}$ ' $S$; $74^{\circ} 49^{\prime} 49^{\prime}$ ' W ) at the Guafo island, Chiloé, Boca del Guafo, Canal Corcovado, on the coast of Xth Region of Chile. The location of Southern sea lion (LMC) and Southern fur seal (LFA) breeding colonies are indicated. 
En esta colonia, establecida en el borde norte y noroeste del cerro de Punta Weather se identifican seis sectores empleados por los lobos marinos tanto para el descanso como la reproducción. En la presente investigación, los sectores muestreados corresponden a aquellos que congregan sobre el $70 \%$ de la población de lobos finos en Punta Weather (i.e., sector $3 \mathrm{y}$ sector $4 ; \mathrm{x}_{\mathrm{S} 3}+\mathrm{S} 4$ dos temporadas $=2249.5 \pm 168.4$ $\mathrm{EE} ; \mathrm{x}$ tamaño poblacional dos estaciones $=3051.8 \pm$ 203.8.4 EE). Las zonas de muestreo presentan áreas con plataformas de arenisca y playas de guijarros de suave pendiente, combinados con zonas rocosas abruptas de tipo conglomerados de cuarzo y granito. Se puede acceder a las distintas zonas por el cerro y por la costa. En ambos sectores se renuevan pozas intermareales durante la marea alta que se mantienen con agua durante gran parte del día. El punto de observación se estableció sobre 20 $\mathrm{m}(\mathrm{S} 3)$ y $50 \mathrm{~m}$ (S4) de altura cubriendo visualmente la mayoría de los animales.

\section{Metodología de muestreo}

Los muestreos fueron realizados durante dos temporadas reproductivas consecutivas, 20042005 y 2005-2006, completando un total de 800 h de observación. Durante la primera temporada se estableció un campamento en forma continua desde el 8 de noviembre de 2004 al 28 de febrero del 2005. En la segunda temporada, el campamento de estableció desde el 10 de diciembre del 2005 al 5 de febrero del 2006 realizándose muestreos entre el 25 al 28 de febrero del 2006. La fecha de inicio de los muestreos del segundo año presentó una demora de dos semanas, producto de los continuos frentes de mal tiempo que imposibilitaron el ingreso a la Isla.

Las observaciones se desarrollaron entre las 08:00 y las 20:00 $\mathrm{h}$ realizando las siguientes actividades, supeditadas a las condiciones meteorológicas imperantes: (a) Durante la identificación de los territorios y determinación de la estructura etaria de los lobos marinos, se registró el número de machos territoriales (i.e., territorios), su fluctuación en el tiempo y la estructura etaria en dos sectores de la colonia (i.e., S3 y S4). Para ello se realizaron semanalmente entre dos a tres conteos por un investigador, de las distintas clases "etarias" o funcionales de los lobos marinos (i.e., hembras, juveniles, cachorros y machos territoriales, VazFerreira 1982, Bianco et al. 1987¹). Estos conteos fueron repetidos cuando ellos presentaron diferencias mayores o igual al $10 \%$. En todos los censos se emplearon un contador manual y binoculares (Samsung 7-15 x35, Samsung $7 \times 25)$. La estructura etaria de cada temporada investigada se obtuvo al promediar las proporciones de machos: hembras: juveniles: cachorros registrados durante el período en que existía el $90 \%$ de los de territorios y el $50 \%$ de los cachorros había nacido (i.e., 15 al 31 de diciembre) (Tabla 1). (b) El nivel de poliginia (i.e., número de hembras reproductoras asociadas a un macho) fue obtenido mediante la aplicación del índice de hembras por macho territorial (HPMT), que describe el número de hembras fértiles con relación a los machos territoriales en el área reproductiva. El número de hembras fértiles se obtiene indirectamente a partir del número máximo de cachorros estimados y el número de machos en función de los territorios establecidos en el sector bajo estudio (Araya et al. $1987^{2}$, Pavés et al. 2005a). (c) Para la determinación del período de partos y cópulas, dos observadores realizaron dos a tres censos diarios del número de cachorros, además registraron la frecuencia de cópulas por hora mediante muestreos ad libitum entre las 08:0020:00 h en los sectores monitoreados (i.e., sector 3 y 4). Tanto a los censos de crías, como las tablas de frecuencia de cópulas se les ajustó una ecuación logística, similar a la metodología descrita por Pavés et al. (2005a) y basado en Trites (1992). Se aplicó esta metodología debido a que mediante la incorporación de datos obtenidos a partir de distintos esfuerzos de muestreo, pueden ser comparados en forma objetiva y con criterios similares, además permite retrocalcular periodos iniciales en ausencia de datos si se cuenta con una detallada

1 BIANCO J, A PONCE DE LEÓN \& R VAZFERREIRA (1987) Interacciones entre machos y cachorros de Otaria flavescens (Shaw), león marino sudamericano, en el Uruguay (Pinnipedia, Otariidae). Anais da $2^{\circ}$ Reuniao de Trabalho de Especialistas em Mamíferos Aqúaticos da América do Sul. Rio Janeiro, Brasil: 24-27.

2 ARAYA H, F CONTRERAS, F CAMPOS, M ARROYO \& E RODRÍGUEZ (1987) Biología reproductiva del león sudamericano (Otaria flavescens) en la lobería de Punta Negra, IquiqueChile. Anais da $2^{\text {a }}$ Reunao de Trabalho de Especialistas em mamíferos Aquaticos da América do Sul. Rio Janeiro, Brasil: 9-16. 
descripción del periodo de incremento exponencial del patrón y del máximo o periodo asintótico de este. La ecuación empleada fue: $P_{t}=A / 1+c e^{-k t}(1)$; donde, $\mathrm{P}_{\mathrm{t}}=$ frecuencia acumulada de cachorros $=$ partos o cópulas $/$ hora en el tiempo "t"; $\mathrm{A}=$ asintótica de los partos/ cópulas; $\mathrm{c}=$ constante de integración; $\mathrm{k}=$ tasa fija de incremento de los partos/cópulas; y, $\mathrm{t}=$ tiempo. En este modelo se ignora el número de crías que mueren entre los conteos consecutivos y considera insignificante los desplazamientos de estas entre la colonia y otras áreas. A partir de los valores estimados de la ecuación (1), se determinó la frecuencia de los partos y cópulas en el tiempo ( $\mathrm{dPartos}_{\mathrm{t}} / \mathrm{dt}$ y dCópulas $/$ dt), graficándose el período de duración y la fecha máxima de estos. La fecha media o máximo de los partos $\left(\mathrm{M}_{\mathrm{p}}\right)$ y cópulas $\left(\mathrm{M}_{\mathrm{c}}\right)$ fueron obtenidas a partir de la fórmula: $M_{p}=[-\ln (1 / c)] / k(2)$; donde, los parámetros empleados en las ecuaciones (2), corresponden a aquellos obtenidos a partir de la ecuación (1) (Trites 1992).

A partir de las curvas de frecuencia de partos y cópulas, se determinó el período de sincronización reproductiva, definida como el período en el cual se registra el $90 \%$ de las pariciones en fechas próximas, y que posee como punto medio la fecha del máximo de ellas (Boness 1991).

\section{Análisis estadístico}

Los análisis estadísticos fueron realizados empleando STATISTICA 6.0 para Windows 98, (Statsoft Inc. 2001). Se realizaron pruebas G (razón log-probabilidad), $\mathrm{G}_{\mathrm{c}}$ (razón logprobabilidad con corrección de continuidad de Yates) y chi-cuadrado $(\chi 2)$ para determinar las posibles diferencias en tablas de contingencia y datos categóricos entre temporadas (e.g., índices de poliginia, proporción etarias). Se usaron pruebas t de Student para comparar el tamaño poblacional entre las temporadas de estudio.

Debido a que el número de territorios, partos y cópulas no presentaron varianza homogénea como tampoco una distribución normal, se aplicaron pruebas no paramétricas para compararlos entre temporadas. Pruebas pareadas de Wilcoxon se usaron para examinar diferencias en el número de territorios, partos y cópulas entre temporadas, mediante una comparación día por día de su número (i.e., compara el número total de cada evento). Pruebas de mediana fueron aplicadas para determinar diferencia en las fechas del máximo de los patrones reproductivos, por medio de una comparación del arreglo de los datos en torno a ella (i.e., compara las fechas del máximo de cada evento) (Zar 1999).

\section{TABLA 1}

Variación del número de territorios y de la estructura etárea observada en la colonia reproductiva de A. australis de Punta Weather, Isla Guafo, Chiloé-Chile, durante las temporadas reproductiva 20042005 y 2005-2006

Age structure variation and number of territories observed during the breeding seasons 2004-2005 and 2005-2006. Number of different age class per territorial male and number of territories are also given for Punta Weather, Isla Guafo, Chiloé-Chile

\begin{tabular}{lcccccccccccccc}
\hline Fecha & MA & M & H & J & C* & 1 & MA & M & H & J & $\begin{array}{c}\text { C* } \\
\text { Valor } \\
\text { de G }\end{array}$ & $\begin{array}{c}\text { Valor } \\
\text { de P }\end{array}$ \\
\hline 8-XI-04 & 69 & 1,0 & 3,1 & 6,5 & 0,0 & - & - & - & - & - & - & - & - \\
26-XI-04 & 239 & 1,0 & 3,1 & 1,4 & 0,1 & - & - & - & - & - & - & - & - \\
3-XII-04 & 251 & 1,0 & 3,9 & 0,2 & 0,3 & - & - & - & - & - & - & - & - \\
16-XII-04 & 256 & 1,0 & 6,0 & 0,2 & 1,9 & $14-X I I-05$ & 221 & 1,0 & 4,9 & 0,3 & 3,1 & 0,435 & $\mathrm{~ns}$ \\
23-XII-04 & 256 & 1,0 & 5,4 & 0,0 & 2,0 & $24-X I I-05$ & 194 & 1,0 & 7,1 & 0,2 & 4,6 & 0,437 & $\mathrm{~ns}$ \\
27-XII-04 & 247 & 1,0 & 6,0 & 0,0 & 3,5 & $28-X I I-05$ & 228 & 1,0 & 6,1 & 0,3 & 3,8 & 0,260 & $\mathrm{~ns}$ \\
5-I-05 & 178 & 1,0 & 7,7 & 0,0 & 6,8 & $2-\mathrm{n}-06$ & 167 & 1,0 & 9,0 & 0,1 & 6,0 & 0,167 & $\mathrm{~ns}$ \\
19-I-05 & 49 & 1,0 & 24,0 & 0,1 & 26,7 & $17-I-06$ & 53 & 1,0 & 25,5 & 0,2 & 21,4 & 0,419 & $\mathrm{~ns}$ \\
\hline
\end{tabular}

MA = Machos adultos totales; $\mathrm{M}=$ machos; $\mathrm{H}=$ hembras; $\mathrm{J}=$ juveniles $; \mathrm{C}=$ cachorros;

* = obtenido desde máximos censales. $\mathrm{n} . \mathrm{s}=$ sin diferencia estadísticamente significativa 


\section{RESULTADOS}

\section{Estructura poligínica en Punta Weather}

La proporción de macho territorial: hembra: juvenil: cachorros registrada en la colonia de Punta Weather fue de 1.0: 5,8: 0,1: 2,5 y 1.0 : 6,0: 0,3: 3,8 para las temporadas 2004-2005 y 2005-2006 respectivamente. No se determinaron diferencias estadísticamente significativas entre temporadas $\left(\chi^{2}=0,257, \mathrm{df}=3 ; \mathrm{P}>0,05\right)$. El número de hembras reproductivas asociadas a un macho territorial varió significativamente entre temporadas (i.e., 2,9 \pm 0.6 HPMT durante 20042005 , y 5,6 \pm 0.5 HPMT durante la segunda; $\mathrm{t}_{4}=$ $-3,553 ; \mathrm{P}=0,023)$. El tamaño poblacional promedio determinado para los meses de diciembre y enero, no presentó diferencias estadísticamente significativas entre las temporadas (temporada 2004/2005, $\mathrm{x}=2.476,8$ $\pm 107,6$ EE; temporada 2005/2006, $x=2.465,0 \pm$ 108 , cuatro ejemplares; $\left.\mathrm{t}_{8}=0,077 ; \mathrm{P}=0,940\right)$.

\section{Territorios de la colonia reproductiva de Punta Weather}

En Punta Weather durante la primera temporada de estudio fue posible identificar entre 0-220 territorios, registrándose el $90 \%$ entre el 3 y 27 de diciembre de 2004. El número máximo de territorios se observó entre el 1 al 15 de diciembre de 2004 ( $n=220$, Fig. 2). Durante la segunda temporada 2005-2006, se registraron entre 0 a 152 territorios, valor que no presentó diferencias estadísticas a lo observado durante la primera temporada (prueba de Wilcoxon, $\mathrm{T}=$ 3,$00 ; \mathrm{Z}=1,213 ; \mathrm{n}=6 ; \mathrm{P}=0,224 ;$ Fig. 2). Se determinó que el $90 \%$ de los territorios estuvo constituido desde inicio del período de muestreo hasta la última semana de diciembre. El número máximo de territorios en los sectores analizados de Punta Weather fue detectado durante la segunda quincena de diciembre del 2005 ( $\mathrm{n}=$ 152; Fig. 2).

Al comparar las dos temporadas, es posible evidenciar que los primeros territorios se establecerían a fines octubre, existiendo un incremento sostenido durante noviembre (i.e., ca 80 territorios cada 15 días), hasta alcanzar su máximo durante la segunda quincena de diciembre; fecha posterior a la cual los machos se retiran y el número de territorios decrece (i.e., ca 40-60 territorios cada 15 días).

\section{Período de partos en Punta Weather}

Durante 2004-2005 y 2005-2006, los parámetros estimados de la ecuación (1) para

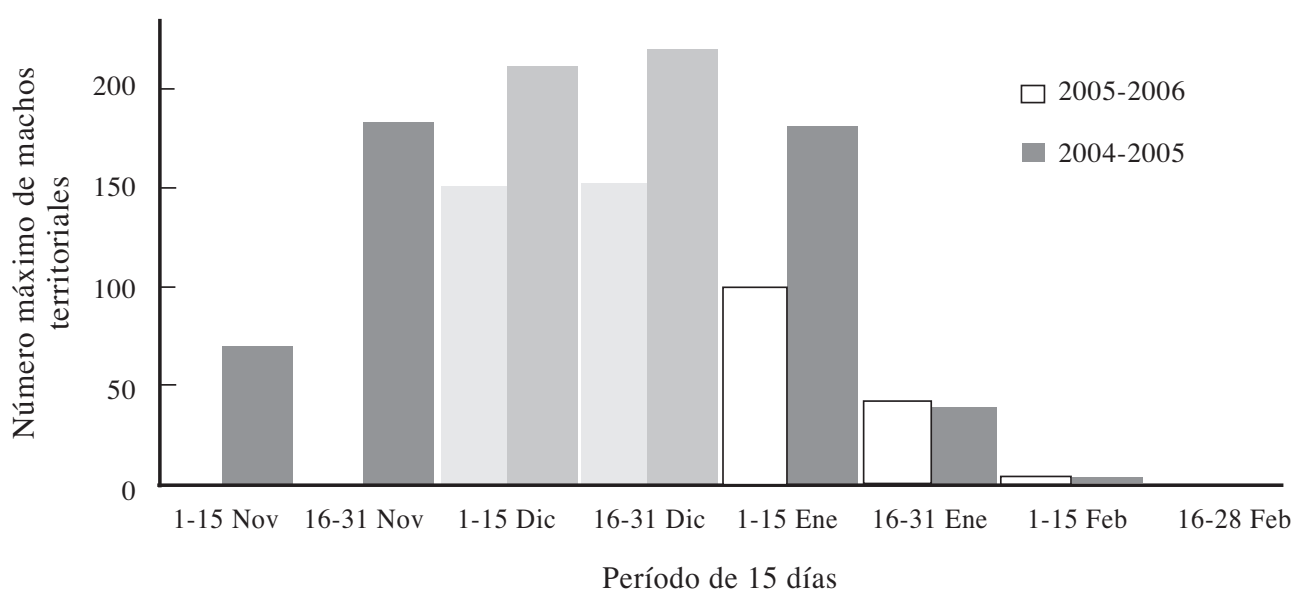

Fig. 2: Variación del número de territorios durante la temporada reproductiva 2004-2005 y 2005 2006 en los sectores reproductivos principales del lobo fino austral en Punta Weather, Isla Guafo, Chiloé, X Región de Chile. Se marca con puntos las quincenas donde se registró el $90 \%$ de los territorios.

Male territory number changes during breeding season 2004-2005 and 2005-2006 on Punta Weather southern fur seal colony, Isla Guafo, Chiloé, X Región de Chile. Bars with spots, with or no black, indicates the period where we observed $90 \%$ of the territorial males in the colony. 
determinar el período de pariciones tuvieron los siguientes valores; $\mathrm{A}=1.320,32, \mathrm{k}=0,20 \mathrm{y} \mathrm{c}=$ $3.279,42$, y $\mathrm{A}=1.130,05, \mathrm{c}=197,62 \mathrm{y} \mathrm{k}=$ 0,22 . Esto indica que para la primera temporada se estimó cerca de 1.300 nacimientos y durante el segundo periodo alrededor de 1.100 nacimientos para los dos sectores evaluados.

El período de pariciones en Punta Weather se identificó en fechas similares durante el período de investigación (Tabla 2). Además, el número de partos por día no fue estadísticamente diferente entre temporada (prueba de Wilcoxon, $\mathrm{T}=796,00 ; \mathrm{Z}=1,806 ; \mathrm{n}$ $=65 ; \mathrm{P}=0,07)$.

Durante la primera temporada el $90,7 \%$ de las pariciones $(\mathrm{n}=1197, \mathrm{~N}=1319)$ fueron registradas entre el 3 de diciembre de 2004 al 1 de enero de 2005, con una duración de 30 días. Por su parte, el período de sincronización reproductiva de la segunda temporada se determinó entre el 1 al 27 de diciembre de 2005, es decir durante 27 días $(90,3 \% ; \mathrm{n}=$ 1014; $\mathrm{N}=1.123$; Tabla 2). La fecha del máximo de pariciones entre temporadas presentó una diferencia de tres días, determinándose entre estas una diferencia estadísticamente significativa (i.e., 17 de diciembre de 2004 y 14 de diciembre de 2005; prueba de medianas, $\chi^{2}=7,723 ;$ df $=1 ; \mathrm{P}=$ 0,005; Tabla 2).

Mediante los datos obtenidos se estima que la fecha media del máximo de partos en Punta Weather correspondió al día 15,5 de diciembre $( \pm 2,12$ día $)$.

\section{Período de cópulas en Punta Weather}

Los parámetros de la ecuación (1) empleados para determinar el período de cópulas durante la primera y segunda temporada fueron los siguientes: $\mathrm{A}=5,17 ; \mathrm{c}=2.009,49, \mathrm{k}=0,18, \mathrm{y}$ $\mathrm{A}=7,00, \mathrm{c}=474,43 ; \mathrm{k}=0,22 . \mathrm{Al}$ igual que lo observado con las pariciones, el período de cópulas se registró en fechas similares entre temporadas, sin evidenciarse diferencias estadísticas en la frecuencia de cópulas/hora entre estas (prueba de Wilcoxon, $\mathrm{T}=880,00 ; \mathrm{Z}$ $=1,790 ; \mathrm{n}=68 ; \mathrm{P}=0,07 ;$ Tabla 2).

TABLA 2

Fecha de ocurrencia de los patrones reproductivos del lobo fino austral durante dos temporadas consecutivas (Punta Weather, X Región-Chile). Se muestra la diferencia en días, porcentaje de variación y valores de $\mathrm{P}$

Reproductive pattern dates for the southern fur seal breeding colony (Punta Weather, X región-Chile). Indication for the difference in days, the variation percentage and $\mathrm{P}$ values are included

\begin{tabular}{|c|c|c|c|c|}
\hline Evento y territorio & 2004-2005 & 2005-2006 & Desfase & Variación $(\%)^{\mathrm{a}}$ \\
\hline Fecha del máximo & $\begin{array}{c}\text { Cuarta semana de } \\
\text { diciembre (23 diciembre) }\end{array}$ & $\begin{array}{c}\text { Cuarta semana de } \\
\text { diciembre ( } 24 \text { diciembre })\end{array}$ & Ninguno & 0 \\
\hline Permanecía del $90 \%$ de MT & Diciembre & Diciembre & Ninguno & 0 \\
\hline Ultimo territorio & Primera semana febrero & Primera semana febrero & Ninguno & 0 \\
\hline Fecha de desintegración & Primera semana enero & Primera semana enero & Ninguno & 0 \\
\hline Primer parto observado & 11 de noviembre & 14 de diciembre. & 34 & +309 \\
\hline Ultimo parto observado & 3 de enero & 2 de marzo & 58 & +1933 \\
\hline Fecha máximo de partos & 17 de dic. & 14 de diciembre & -3 días & $-21,4$ \\
\hline $\begin{array}{l}\text { Sincronización de las } \\
\text { pariciones (90\% en días) }\end{array}$ & $\begin{array}{l}3 \text { de diciembre al } \\
1 \text { de enero (30) }\end{array}$ & 1 al 27 de diciembre (27) & - (3 días) & $-(+11,1)$ \\
\hline Primera cópula observada & 12 de noviembre & 16 de diciembre & 34 días & +283 \\
\hline Ultimo cópula observada & 24 de enero & 9 de enero & 15 día & $+62,5$ \\
\hline Fecha máximo de cópulas & 18 de diciembre & 20 de diciembre & 2 días & +11 \\
\hline $\begin{array}{l}\text { Sincronización de las cópulas } \\
\text { (90\% en días) }\end{array}$ & $\begin{array}{l}3 \text { de diciembre al } \\
3 \text { de enero (32) }\end{array}$ & $\begin{array}{c}8 \text { de diciembre al } \\
2 \text { de enero (26) }\end{array}$ & $\begin{array}{c}\text { (-6 días) } \\
-\end{array}$ & $-(-18,8)$ \\
\hline
\end{tabular}

${ }^{a}=$ se entrega el porcentaje de variación entre temporadas, signo negativo indica la existencia de una disminución, signo positivo hace referencia a un incremento. 
Se determinó una diferencia de seis días en el período de sincronización de las cópulas entre temporadas, sin ser estadísticamente diferentes (Tabla 2). Durante la primera temporada el 90,4\% de las cópulas $(\mathrm{n}=4,64$ cópulas $\mathrm{h}^{-1}, \mathrm{~N}=5,17$ cópulas $\mathrm{h}^{-1}$ ) fueron identificadas entre el 3 de diciembre y 3 de enero, i.e., en 32 días. Durante la segunda temporada 90,4 \% de las cópulas $(\mathrm{n}=6,31$ cópulas $\mathrm{h}^{-1}, \mathrm{~N}=6,98$ cópulas $\mathrm{h}^{-1}$ ) fueron identificadas entre el 8 de diciembre al 2 de enero, i.e., en 26 días. La fecha del máximo de cópulas presentó un desfase de dos días entre temporadas (i.e., 18 de diciembre de 2004 y 20 de diciembre de 2005), sin ser esta diferencia estadísticamente significativa (prueba de medianas, $\left.\chi^{2}=1,575 ; \mathrm{df}=1 ; \mathrm{P}=0,209\right)$. Mediante el método logístico se estimó que la fecha media del máximo de cópulas en Punta Weather fue el día 19 de diciembre $( \pm 1,41$ días).

La diferencia entre la fecha del máximo de pariciones y del máximo de cópulas permite determinar el inicio del período de estro. De esta forma, durante la primera temporada las hembras parteras fueron copuladas un día después del parto, período inferior al determinado para la segunda temporada (i.e., 6 días postparto).

\section{DISCUSIÓN}

Al comparar el tamaño poblacional promedio de los lobos finos de Isla Guafo, la estructura etaria y el índice de poliginia -HPMT- entre temporadas, solo se registraron diferencias estadísticamente significativas en este último, situación probablemente relacionada con la disminución del $30 \%$ de los machos territoriales entre temporadas, evidenciando una mayor sensibilidad de este parámetro dentro de la dinámica poblacional. Debemos considerar que los datos que permiten calcular los índices de poliginia son a su vez empleados en la determinación del tamaño efectivo poblacional (Oliveira et al. 2006) y por tanto, modificaciones en este índice tienen grandes implicancias en la determinación de la tendencia poblacional y en aspectos evolutivos de la especie (Olivera et al. 2006).

Por otro lado, si comparamos los valores promedios de la estructura etaria (i.e., x 2003/04-
2005/06 $=1$ macho: 5,9 hembras: 0,2 juveniles: 3,2 cachorros) y el índice de poliginia de A. australis (i.e., $x$ 2003/04-2005/06 $=4,2$ HPMT), con aquellos descritos para otras especies de Otariidae, es posible observar que junto a $O$. flavescens, A. galapagoensis y A. tropicalis son los que presentan menor valor (Boness 1991). Además, los valores de poliginia obtenidos en este estudio, tanto en función de las hembras reproductoras y machos territoriales (i.e., HPMT), como en relación con el numero de hembras y machos totales (i.e., estructura etaria) fueron menores a lo descrito para la misma especie en Perú (i.e., 12 - 21 hembra/ macho; Majluf 1987) y en Uruguay (i.e., 9,6 HPMT; Franco 2005).

En otáridos se observa una positiva relación entre el dimorfismo sexual, medido en función del tamaño corporal, y el grado de poliginia (Boness 1991). El marcado dimorfismo potencia la capacidad de los machos de estas especies para acceder, controlar y copular un mayor número de hembras, al mismo tiempo de lograr defender su territorio frente al ingreso y ataques de machos intrusos (Riedman 1990). De esta forma, machos de mayor tamaño podrían controlar una elevada cantidad de hembras, incrementando con ello el nivel de poliginia (Majluf 1987). Sin embargo, debemos considerar, como lo explica Boness (1991) que existen otros factores relacionados con el grado de poliginia en los Otariidae, e.g., efecto ecológico del macho marginal, la topografía abrupta, el espacio disponible y las condiciones meteorológicas. En este sentido, en Isla Guafo en comparación con las colonias de Perú y Uruguay, el efecto ecológico de machos periféricos sobre las agrupaciones reproductivas son despreciables tanto a nivel intraespecífico como interespecífico (observación personal.), condición que disminuiría la agregación de las hembras en torno a un macho dominante protector (Cassini 2000). A su vez, la topografía de las playas en Isla Guafo y el mayor espacio disponible para el establecimiento de las agrupaciones reproductivas también permitirían entender el menor número de hembras por machos y con ello la identificación de un menor nivel de poliginia entre los Otariidae.

En Punta Weather de Isla Guafo, aunque el número y la estacionalidad de los territorios no presentaron diferencias estadísticas entre 
temporadas, se observó en estos una disminución del $30 \%\left(\mathrm{n}_{2004-2005}=220\right.$ territorios, $\mathrm{n}_{2005-2006}=152$ territorios). Cabe destacar que las fechas donde se determinó el máximo número de territorios $(\mathrm{n}=220,23$-XII2004; $\mathrm{n}=152,24-\mathrm{XII}-2005)$ coincidió con aquellas en las cuales se identificó el máximo de cópulas, situación que demuestra una estrecha relación entre la presencia de los machos territoriales y el periodo de apareamiento. Los machos territoriales permanecerían defendiendo su recursoterritorio hasta que la mayor cantidad de hembras hubiese sido copulada, después de lo cual, abandonarían sus áreas para iniciar los viajes de alimentación. Esto evidencia el mayor interés que tiene el macho por aparearse en desmedro del cuidado parental, condición ampliamente registrada en los Otariidae y en especies poligínicas en general (Riedman 1990, Boness 1991).

Entre los patrones utilizados para describir la temporada reproductiva de A. australis en Isla Guafo, el día del máximo de partos, cópulas y el periodo de sincronización reproductiva fueron registradas en fechas similares entre temporadas. Pese a esto, únicamente la fecha del máximo de pariciones presentó diferencias estadísticamente significativas entre estaciones evaluadas (i.e., desfase de tres días). En este sentido, es necesario plantearse si esta diferencia tiene una implicancia biológica para la población o especie, y si por ello es ¿biológicamente significativa? Una implicancia en este contexto tendría mayor repercusión, si ella se constituyen en una barrera de aislamiento reproductivo pericigótico, específicamente del tipo temporal (Futuyma 1997). El desfase de tres días en una misma, o en poblaciones distantes, no generaría tal barrera reproductiva, por tanto no debería ser considerada biológicamente significativo para la especie.

La alteración en la fecha de las pariciones registrado en Isla Guafo puede ser explicado por ingreso a la actividad reproductiva de hembras primerizas donde su proporción, en relación con las hembras de mayor edad que llegan a parir primero, alteraría constantemente la fecha del máximo de partos (Trites 1992). Por su parte, la diferencia identificada en la fecha del máximo de las cópulas en Isla Guafo, estaría directamente asociado al adelantamiento o retraso de la fecha del máximo de los nacimientos entre temporadas, condición común a otras especies de otáridos (Campagna 1985, Goldsworthy \& Shaughenessy 1994).

Tanto el período de sincronización de las pariciones como el de las cópulas, fechas en las cuales se registra el $90 \%$ de estas (Boness 1991), presentaron una duración similar entre temporadas (i.e., alrededor de 30 días). En estos parámetros, fue posible determinar una diferencia de +3 días y -6 días respectivamente, sin ser ello de significancia estadística. En ambos casos, las pariciones y copulas fueron observadas en el mes de diciembre, siendo la fecha del máximo promedio el día 16 de diciembre para los partos (i.e., 17 y 14 de diciembre de 2004) y el 19 de diciembre para las cópulas (i.e., 18 y 20 de diciembre de 2005).

La estabilidad en las fechas en que se registraron los períodos reproductivos en Isla Guafo, también ha sido observada en otros Otariidae, siendo esta una característica marcada y generalizada del grupo, situación íntimamente relacionada con la rigidez del ciclo reproductivo de estos animales (Campagna 1985, Araya et al. 1986, Duck 1990, Riedman 1990, Boness 1991, Goldsworthy\& Shaughnessy 1992, Majluf 1992, Trites 1992, Acevedo et al. 2003, GarciaAguilar \& Aurioles-Gamboa 2003, Pavés et al. 2005a).

Con los datos presentados en este estudio, se estimó que el ciclo reproductivo del lobo fino austral en Isla Guafo, determinado entre la fecha máxima de las cópulas de un año y fechas del máximo de las pariciones del siguiente, presentaría una duración de 11 meses 27 días, período que se encuentra dentro del rango indicado para los Otariidae (Costa 1991). A su vez, esta información permitiría indicar una diapausa embrionaria de dos meses 27 días, considerando los nueve meses restantes empleados para la gestación embrionaria (Costa 1991) y postulando a su vez, que el periodo de implantación embrionaria en este sector se identificaría durante el mes de marzo de cada año.

Al realizar una comparación en las fechas de pariciones y estacionalidad de los territorios entre poblaciones distantes de A. australis de América del Sur, se evidencia un conjunto de diferencias. En Punta San Juan (Perú; $15^{\circ} 22^{\prime}$ S, 
$75^{\circ} 12^{\prime} \mathrm{W}$ ), el período de pariciones se registra en el mes de noviembre, con fecha del máximo promedio el día 15 de noviembre (i.e., 11 y 19 de noviembre de 1984 y 1985, Majluf 1987), un mes antes de lo observado en Isla Guafo. El período de sincronización de estas por su parte, presenta una duración mayor a lo observado en Isla Guafo (i.e., 38 y 41 días de duración en Punta San Juan). Igualmente, aunque machos territoriales en ambas localidades son observados durante tres meses (i.e., Punta San Juan oct-nov-dic; Isla Guafo, nov-dic-ene), las fechas en que se identifican están desplazadas también un mes. Además, el $85 \%$ de los territorios están constituidos entre el 25 de noviembre y el 14 de diciembre en Punta San Juan, registrándose el máximo el 7 de diciembre, i.e., tres semanas antes de lo observado en Isla Guafo.

Al igual que lo registrado por este estudio, y pese a estas diferencias en la estacionalidad de los territorios y pariciones identificado entre colonias, la fecha del máximo de machos territoriales en Punta San Juan se identifica aproximadamente dos semanas después del máximo de partos, coincidente con el período de cópula y previo a la retirada de los machos de sus territorios, muy similar a los lobos finos de Isla Guafo.

Por su parte, en Isla de Lobos (Uruguay; $35^{\circ} 01^{\prime} \mathrm{S}, 54^{\circ} 50^{\prime} \mathrm{W}$ ), el máximo de pariciones y de cópulas fueron identificados el 10 de diciembre y el 14 de diciembre de 1991 respectivamente (Pavés et al. 2005b ${ }^{3}$ ). Esta situación evidencia que los datos registrados en Isla de Lobos (Uruguay) presentan mayor similitud a los obtenidos en Isla Guafo (Chile) que a los publicados para Punta San Juan (Perú). Sin embargo, se hace necesario prontamente, comparar datos actuales para determinar tanto la permanencia de esta tendencia o la existencia de diferencias entre ellas. Estas diferencias identificadas en tres poblaciones de América del Sur, donde los eventos reproductivos son observados un mes antes entre las poblaciones del Perú, de aquellos presentes en Chile y Uruguay, podrían

\footnotetext{
PAVÉS H, R SCHLATTER, M BATALLÉS \& L CAPPOZZO (2005b) Sincronización reproductiva del lobo fino austral, Arctocephalus australis, en América del Sur. Libro de resúmenes de las XX Jornadas de Mastozoología Argentina, 8-11 de noviembre, Buenos Aires, Argentina.
}

tener implicancias biológicas y sistemáticas significativas. Se ha mencionado la existencia de tres agrupaciones morfológicamente distintas de A. australis (i.e., norte de ChilePerú, sur de Chile y Atlántico; Oliveira et al. 1999, 2005a) y de al menos dos grupos genéticamente bien separadas (i.e., norte de Chile-Perú y Atlántico; Márquez et al. 20034 Oliveira et al. 2005b ${ }^{5}$, Túnez et al. 2006). Según estos autores, estos hechos evidenciarían la existencia de historias evolutivas distintas entre estas poblaciones, modulado por la presencia de barreras de aislamiento reproductivo pericigóticas (i.e., tipo espacial por la distribución fragmentada) que permitirían identificar al menos dos unidades evolutivas independientes, como lo sugiere Oliveira et al. $\left(2002^{6}, 2005 a, 2005 b\right)$. En este contexto, la capacidad de identificar diferencias estadísticamente significativas en la estación reproductiva, en el periodo de pariciones, cópulas o territorios entre años o entre poblaciones tendría una significancia biológica si se logra acreditar la presencia de unidades evolutivas independientes.

Pese a la diferencia significativa registrada en el periodo de sincronización de pariciones en Isla Guafo, la fecha del máximo y la duración de la sincronización entre temporadas se observan durante el mismo período de tiempo, indicando una alta estabilidad en esta conducta o "patrón reproductivo", característica bastante marcada en especies de ambientes

\footnotetext{
4 MÁRQUEZ A, J BIANCO, E MALDONADO \& S GONZÁLEZ (2003) Variabilidad genética de los lobos marinos (Arctocephalus australis y Otaria byronia) de Uruguay. Libro de resúmenes de las II Jornadas de Conservación y Uso Sustentable de la Fauna Marina, 1-3 de octubre de 2003, Montevideo, Uruguay.

5 OLIVEIRA L, E HINGST-ZAHER, P MAJLUF, J HOFFMAN, I MORENO, D RODRÍGUEZ, M GOODDAL, W SIELFED, $M$ MUELBERT, E CRESPO, C VENEGAS, W AMOS \& J MORGANTE (2005b) Lobo-marinho sul-americano, uma ou duas espécies? Análises moleculares e morfológicas para a definacao do status taxonómico de Arctocephalus australis (Mammalia, Carnivora, Otariidae). Libro de resúmenes de las III Jornadas de Conservación y Uso Sustentable de la Fauna Marina, 14-17 de septiembre de 2005, Montevideo, Uruguay.

6 OLIVEIRA L, E HINGST-ZAHER, J HOFFMAN, P MAJLUF, W AMOS \& J MORGANTE (2002) Variacao geográfica do lobo-marinho sul americano Arctocephalus australis integracao da analise molecular e morfológica. Libro de resúmenes de la 10 RT y Cuarto Congreso SOLAMAC, 14-19 de octubre de 2002, Valdivia, Chile. 43 pp.
} 
polares y templados (e.g., Otaria flavescens, Arctocephalus australis, Arctocephalus gazella, Callorhinus ursinus, Zalophus californianus; Campagna 1985, Boness 1991, Majluf 1992, Trites 1992, García-Aguilar \& AuriolesGamboa 2003). Sin embargo, en presencia de perturbaciones drásticas ambientales, como aquellas provocadas por el desarticulamiento de las cadenas tróficas durante los ENSO, se generarían marcadas alteraciones de estos patrones principalmente moduladas por los abortos y nacimientos prematuros (e.g., $O$. flavescens: Sielfeld \& Guzmán 2002, Soto et al. 2004; Zalophus californianus: Trillmich \& Limberger 1985, Boness et al. 1991, Ono \& Boness 1991). De modo que, la estabilidad de la temporada reproductiva y la sincronización en estas especie, así como también para los ejemplares de A. australis establecidos en Isla de Guafo, estaría relacionada con la rigidez de su ciclo reproductivo, ciclo que sería el resultado de una fuerte presión de selección natural sobre la descendencia y los progenitores de la especie (Testa 2002).

Los factores ambientales que modularían el ciclo reproductivo de especies de ambientes de climas templados y polares, tienen relación con la marcada estacionalidad climática y alimentaria, lo cual posibilitaría la presencia de condiciones ambientales adecuadas para la supervivencia y desarrollo de la descendencia (Riedman 1990, Boness 1991, Majluf 1992, Trites \& Antonelis 1994, Soto et al. 2004). En este sentido, se ha logrado determinar ciertas relaciones entre aspectos de la biología reproductiva de las especies y las condiciones ambientales, medidas en función de la latitud (Boness 1991). Se ha determinado una relación inversa entre la latitud donde se establecen las poblaciones/especies y la duración del período de sincronización reproductiva, donde aquellas poblaciones o especies establecidas a menor latitud presentarían un período de sincronización de mayor duración (Boness 1991).

Aunque a medida que se incrementa el conocimiento de los patrones reproductivos en Otariidae y Phocidae de amplia área de distribución latitudinal, se ha logrado identificar un conjunto de especies que cumplen con las predicciones del modelo de Boness (e.g., Zalophus californianus wollebaek y Halichoerus gryptus: Riedman 1990; Arctocephalus fosteri: Goldsworthy \&
Shaughgnessy 1992; Phoca groenlandica: Stenson et al. 1993; Zalophus californianus y Phoca vitulina richardsi: Temte 1993; Eumetopias jubatus: Pitcher et al. 2001; Zalophus californianus californianus: GarciaAguilar \& Aurioles-Gamboa 2003). Arctocephalus australis en América del Sur, según la literatura comparada con los resultados de Isla Guafo, pareciese no ajustarse a este modelo, siendo por ello necesario actualizar la información reproductiva de la especie en gran parte de su área de distribución para lograr conclusiones al respecto.

Mediante la información obtenida a través de la presente investigación, y pese a la diferencia estadísticamente significativa registrada en las pariciones, fue posible determinar una marcada estacionalidad y sincronización reproductiva en la especie. Además, el periodo de pariciones, cópulas y la estacionalidad de los territorios presentaron una estabilidad temporal en Isla Guafo, condición compartida con el resto de otáridos de ambientes templados y polares actualmente estudiados. Por último, mediante la comparación entre los resultados obtenidos aquí, con aquellas presentados de otras colonias en América del Sur, se evidencian similitudes y diferencias que necesitan con urgencia ser actualizadas para entender, a la luz de las herramientas filogeográficas la historia evolutiva y ecológica de esta especie.

\section{AGRADECIMIENTOS}

Agradecemos especialmente la ayuda entregada por voluntarios en terreno durante la realización de esta investigación, Claudio Vera Cárdenas, Diego Miranda, Carola Vaccaro, Paula Antileo, Isabel del Moral, Victoria Riquelme, Felipe Henríquez, Felipe Moreno, todos de la Universidad Austral de Chile, y a Sabrina Riveron de la Universidad de la República del Uruguay. Al Dr. Claudio Campagna (Centro Nacional Patagónico), Valentina Franco y Alfredo Lebas (Universidad de la República del Uruguay), Dr. Luis Cappozzo (Museo Argentino de Ciencias Naturales), Dra. Patricia Majluf y Susana Cárdenas (Universidad Cayetano de Heredia), José Carlos Márquez (Instituto del Mar del Perú), Karim Soto (Universidad Cayetano de 
Heredia) y a la Dra. Larissa Oliveira (Universidade Federal Do Rio Grande Do Sul) por la literatura facilitada. Este estudio fue apoyado por el Instituto de Zoología de la Universidad Austral de Chile (UACh), Dirección de Investigación y Desarrollo de la UACh (Proyecto DID-D-2004-7/ Proyecto D2005-15), por la Dr. Cheryl Baduini (The Claremont College, California, USA), la Armada de Chile, Gobernador Marítimo de la X Región, Capitán del Distrito Naval Chiloé, Jefe Centro Zonal Señalización Marítima y la Dotación del Faro de la Isla Guafo y en especial a los Jefe de Faros, Sargentos Muñoz, Guzmán, Oyadenel, de la Jara y Aguayo (20042005/2005-2006), Patrones de las embarcaciones artesanales "Nicole" y "Costamar II". H.J.P. es actualmente estudiante del programa de Doctorado en Ciencias UACh $\mathrm{y}$ ha sido apoyado con beca de MECESUP (AUS0111 y UCO0214), Chile.

\section{LITERATURA CITADA}

ACEVEDO J, A AGUAYO-LOBO \& W SIELFELD (2003) Eventos reproductivos del león marino común Otaria flavescens (Shaw 1800), en el norte de Chile (Pacífico suroriental). Revista de Biología Marina y Oceanografía (Chile) 38: 69-75

ARAYA H, M ARROYO, F CAMPOS \& F CONTRERAS (1986) Conducta reproductiva del lobo marino común (Otaria flavescens) Iquique-Chile. 19841985. Memoria para optar al título Profesor de Estado en Biología y Ciencias. Universidad Arturo Prat, Facultad de Ciencias de la Educación, Iquique, Chile. $165 \mathrm{pp}$.

BONESS D (1991) Determinants of mating system in the Otariidae (Pinipedia). En: Deane Renouf (ed) Behaviour of pinniipeds: 1-35. Chappman and Hall Ltd., New York, New York, USA.

BONESS D, O OFTEDAL \& K ONO (1991). The effect of El Niño on pup development in the California sea ion (Zalophus californianus) Early postnatal growth. En: Billings WD, F Golley, OL Lange, JS Olson \& H Remmort (eds) Pinnipeds and El Niño: responses to enviromental stress: 173-179 SpringerVerlag, Berlin, Germany.

BOWEN W (1991) Behavioural ecology of pinniped neonates. In: Deane Renouf (ed) Behaviour of pinniipeds: 66-128. Chapman and Hall Ltd., New York, New York, USA.

CAMPAGNA C (1985) The breeding cycle of the southern sea lion (Otaria byronia). Marine Mammals Science 1: 210-218.

CAMPAGNA C, M LEWIS \& R BALDI (1993) Breeding biology of southern elephant seals in Patagonia. Marine Mammals Science 9: 34-37.

CASSINI M (2000) A model on female breeding dispersion and the reproductive system of pinnipeds. Behavioural Processes 51: 93-99.

COSTA D (1991) Reproductive and foranging energetics of pinnipeds: implications for life history patterns. En: Deane Renouf (ed) Behaviour of pinniipeds: 300-344. Chappman and Hall Ltd., New York, New York, USA.

COSTA D \& N GALES (2003) Energetics of a benthic diver: seasonal foraging ecology of the Australian sea lion, Neophoca cinerea. Ecological Monographs 73: $27-43$

DUCK D (1990) Annual variation in the timing of reproduction in Antarctic fur seals, Arctocephalus gazella, at Bird Island, South Georgia. Journal of Zoology (London) 222: 103-116.

FAO (1993) Species identification guide marine mammals of the world: Otariidae eared seals. FAO, $232 \mathrm{pp}$.

FRANCO V (2005) Comportamiento maternal y aspectos reproductivos de Arctocephalus australis en isla de Lobos-Uruguay. Tesis de Licenciatura, Universidad de la República, Facultad de Ciencias, Montevideo, Uruguay. $74 \mathrm{pp}$.

FUTUYMA D (1997) Evolutionary biology. Third edition. Sinauer Associates, Sunderland, Massachusetts, USA. $702 \mathrm{pp}$

GARCÍA-AGUILAR M \& D AURIOLES-GAMBOA (2003) Breeding season of the California sea lion (Zalophus californianus) in Gulf of California, México. Aquatic Mammals 29: 67-76.

GOLDSWORTHY S \& P SHAUGHNESSY (1994) Breeding biology and haul-out pattern of the New Zealand fur seal, Arctocephalus forsteri, at Cape Gantheaume, south Australia. Wildlife Research 21: 365-376

MAJLUF P (1987) Reproductive ecology of female South American fur seals at Punta San Juan, Perú. Ph.D Dissertation, University of Cambridge, Zoology Department, Cambridge, United Kingdom. 126 pp.

MAJLUF P (1992) Timing of births and juvenile mortality in South American fur seal in Perú. Journal of Zoology (London) 227: 367-383.

OLIVEIRA L, L MALABARBA \& P MAJLUF (1999) Variação geográfica em crânios do lobo-marinho sul-americano Arctocephalus australis (Zimmermann, 1783) das populações do Brasil e Peru. Comunicações do Museu de Ciências e Tecnologia da PUCRS 12: 179-192.

OLIVEIRA L, E HINGST-ZAHER \& J MORGANTE (2005a) Size and shape sexual dimorphism in the skull of the South American fur Seal, Arctocephalus australis (Zimmermann, 1783) (Carnivora: Otariidae). Latin American Journal of Aquatic Mammals 4: 27-40.

OLIVEIRA L, M ARIAS-SCHREIBER, D MEYER \& J MORGANTE. (2006) Effective population size in a bottlenecked fur seal population. Biological Conservation 131: 505-509.

ONO K \& D BONESS (1991) The influence of el Niño on mother-pup behavior, pup ontogeny, and sex ratios in the California sea lion. En: Billings WD, F Golley, OL Lange, JS Olson \& H Remmert (eds) Pinnipeds and El Niño: responses to enviromental stress: 185-192. Springer-Verlag, Berlin, Germany.

PAVES H, R SCHLATTER \& C ESPINOZA (2005a) Patrones reproductivos del lobo marino común, Otaria flavescens (Shaw 1800), en el centro-sur de Chile. Revista Chilena de Historia Natural 78: 673686.

PITCHER K, V BURKANOV, D CALKINS, B LEBOEUF, E MAMAEV, $R$ MERRICK \& G PENDLETON (2001) Spatial and temporal variation in the timing of births of steller sea lions. Journal of Mammalogy 82: 1047-1053. 
RIEDMAN M (1990) The pinnipeds: seals, sealions, and walruses. University of California Press, Berkeley, California, USA. 439 pp.

SIELFELD W (1983) Mamíferos marinos de Chile. Ediciones de la Universidad de Chile, Santiago, Chile. 199 pp

SIELFELD W (1999) Estado del conocimiento sobre conservación y preservación de Otaria flavescens (Shaw 1800) y Arctocephalus australis (Zimmerman 1783) en las costas de Chile. Estudios Oceanológicos (Chile) 18: 81-96.

SIELFELD W \& A GUZMAN (2002) Effect of El Niño $1997 / 98$ on a population of the southern sea lion (Otaria flavescens Shaw) from Punta Patache/Punta Negra (Iquique, Chile). Investigaciones Marinas (Chile) 30: 157-160.

SOTO K, A TRITES \& M. ARIAS-SCHREIBER (2004) The effects of prey availability on pup mortality and the timing of birth of South American sea lions (Otaria flavescens) in Perú. Journal of Zoology (London) 264: 419-428.

STENSON G, R MYERS, M HAMMILL, NI I-H, W WARREN \& M KINGSLEY (1993) Pup production of Harop seals, Phoca groenlandica, in the northwest Atlantic. Canadian Journal Fisher and Aquatic Science 50: 2429-2439.

STATSOFT INC. (2001) Statistica (data análisis software system, Versión 8. www.statsoft.com).

TEMTE J (1993) Latitudinal variation in the birth timing of captive California sea lions and other captive north Pacific pinnipeds. Fishery Bulletin 91: 710717.

TESTA J (2002) Does predation on neonates inherently select for earlier birth? Journal of Mammalogy 83: 699-706.
TORRES J \& L CONTRERAS (1987) Presencia del lobo fino austral (Arctocephalus australis) en Isla Guafo, Chiloé. Informe Final, Proyecto FONDECYT 86/ 1461, 4 pp. Fuente electronica: http:// www.conicyt.cl/bases/bibfon/6/1/861461.html

TRILLMICH F \& D LIMBERGER (1985) Drastic effects of El Niño on Galapagos pinnipeds. Oecologia 67: 19-22.

TRITES A (1992) Reproductive synchromy and the estimation of mean date of birth from daily counts of Northern fur seal pups. Marine Mammal Science 8: 44-56.

TRITES A \& G ANTONELIS (1994) The influence of climatic seasonality on the life cycle of the Pribilof northern fur seal. Marine Mammal Science 10: 311324.

TÚNEZ I, D CENTRO, L CAPPOZZO \& M CASSINI (2006) Geographic distribution and diversity of mitochondrial DNA haplotypes in South American sea lions (Otaria flavescens) and fur seals (Arctocephalus australis). Mammalian Biology 72: 193-203.

VAZ-FERRERIA R (1965) Ecología terrestre y marina de los pinnipedios del Atlántico Sudoccidental. Anais da Academia Brasileira de Ciencias (Brasil) 137: 179-191.

VAZ FERREIRA R (1982) Mammals in the seas. FAO Fisheries Series 5, Volume 4. Small cetaceans, seals, sirenias and otters. $531 \mathrm{pp}$.

ZAR JH (1999) Biostatistical analysis. Fourth edition. Prentice Hall, Upper Saddle River, New Jersey, USA. 663 pp.

Editor Asociado: Juan Carlos Torres-Mura

Recibido el 22 de septiembre de 2006; aceptado el 21 de Agosto 2007 
